Www.jmscr.igmpublication.org

Impact Factor 5.84

Index Copernicus Value: 83.27

ISSN (e)-2347-176x ISSN (p) 2455-0450

crossref DOI: _https://dx.doi.org/10.18535/jmscr/v5i3.144

Journal Of Medical Science And Clinical Research

IGM Publication

An official Publication of IGM Publication

\title{
Original Research Article \\ An Etiological Study of Acute Kidney Injury in Patients at Tertiary Care Hospital in Navi Mumbai
}

\author{
Authors \\ Kejriwal $\mathbf{A}^{\mathbf{1}}$, Naga $\mathbf{P}^{2}$, Desai $\mathbf{H}^{3}$ \\ ${ }^{1}$ Professor, Department of General Medicine, MGMIHS, Navi Mumbai \\ ${ }^{2,3}$ Resident, Department of General Medicine, MGMIHS, Navi Mumbai
}

\begin{abstract}
Aims and Objective: To determine the etiology of acute kidney injury

Material and Method: In our study 100 patients admitted in MGM hospital in medical ward and MICU with diagnosed AKI were enrolled. A detailed history was obtained, detailed systemic examination followed by relavant investigations were conducted and result noted.

Result: In 100 patients maximum age group involved is 41-50years(41\%),common etiologies are multi organ failure with sepsis $(16 \%)$ followed by snakebite(12\%), malaria(10\%), congestive cardiac failure(9\%), urinary tract obstruction(8\%), cholera(6\%), myocardial infarction(6\%),dengue(5\%), Acute exacerbation of COPD and acute gastroenteritis(4\%), while remaining etiologies constituted $20 \%$ of etiological factors.

Conclusion: Acute kidney injury is an increasingly common and potentially catastrophic complication. Mortality rates remain unacceptably high despite significant advance in health care. The diagnosis of AKI is based on indirect measures of GFR, plasma urea and creatinine concentrations. AKI can be prevented if detected early and appropriate treatment is initiated early.

Key Words: Acute Kidney Injury, Multi-organ failure, Sepsis.
\end{abstract}

\section{Introduction}

Definition: Acute Kidney Injury (AKI) is a clinical syndrome characterized by rapid (hours to weeks) decline in glomerular filtration rate (GFR) and retention of nitrogenous waste products such as blood urea nitrogen (BUN) and serum creatinine with perturbation of extracellular fluid volume and electrolyte and acid base homeostasis ${ }^{(1,2)}$.

Nissenson defined AKI as "Rapid decline in glomerular filtration rate resulting from direct ischemic or toxic injury to kidney" occurs due to reduction in glomerular capillary permeability, back leak of glomerular filtrate, tubular obstruction and intra renal vasoconstriction ${ }^{(3)}$.
The kidney being relatively unique among organs of the body in its ability to recover from almost complete loss of function. Acute kidney injury is responsible for major morbidity and mortality of hospitalized individuals because of serious nature of underlying illness and high incidence of complications. It is rare for a patient to die directly as a result of acute kidney injury, but is an independent risk factor for death ${ }^{(4,5,6)}$. Factors other than loss of renal functions like bleeding and sepsis will determine the outcome and their identifyication is necessary to improve the prognosis of acute kidney injury ${ }^{(1)}$. 
Acute kidney injury is a common clinical condition in hospitalized patients ${ }^{(7)}$ with mortality rates varying between $10-80 \%{ }^{(8)}$. Acute kidney injury can be hospital acquired or community acquired. The most common causes of Hospital acquired Acute Kidney Injury are due to medical causes mainly drug induced followed by sepsis (ICU). The most common causes of Community acquired Acute Kidney Injury are acute diarrheal illness, malaria, sepsis, leptospirosis, snake bite followed by obstetric and surgical causes. Most common clinical features are oliguria (82.5\%) followed by encephalopathy, vomiting and $\operatorname{acidosis}^{(7)}$.

Mortality in patients with uncomplicated AKI- 10 $\%(9,10)$.

Mortality in patients with AKI and multi organ failure- $50 \%$.

Mortality inpatients who require renal Replacementtherapy- $80 \%{ }^{(11,12)}$

The diagnosis of AKI in clinical practice is most often based on indirect measures of GFR, plasma urea and creatinine concentrations, and infrequently also on the clearance of creatinine over a 24-hour urine collection. Recently, a novel marker of renal function, cystatin $\mathrm{C}$, was proposed to be more sensitive than plasma creatinine for various forms of chronic renal dysfunction ${ }^{(13)}$.

The present study was undertaken to determine the etiology of acute kidney injury

\section{Material and Method}

Source of Data: The present study, an observational study was conducted in MGM college and Hospital, a tertiary care hospital in Navi Mumbai, Maharashtra. All patients who were having met with AKIN criteria were included.

Duration of study: From October 2013 to July 2016.

Study Design: Prospective observational study of 100 cases. All patients studied with a detailed history, physical examination, systemic examination and investigated.

\section{Inclusion Criteria}

- Patients admitted in medical ward and MICU with clinical and biochemical evidence of acute kidney injury.

- Age group between 16-60 years

\section{Exclusion Criteria}

- Patients below age group of 16 years and above age group of 60 years

- Patients who were diagnosed as acute kidney injury and progressed to chronic kidney disease on follow up

- Patients admitted in surgery and OBGY ward

\section{Results}

Out of 100 patients maximum number of patients belonged to age group 41-50 years, while 23 patients belonged to 51-60 years and there were only 2 patients below 16-20 years. Mean age was 43.5 years.

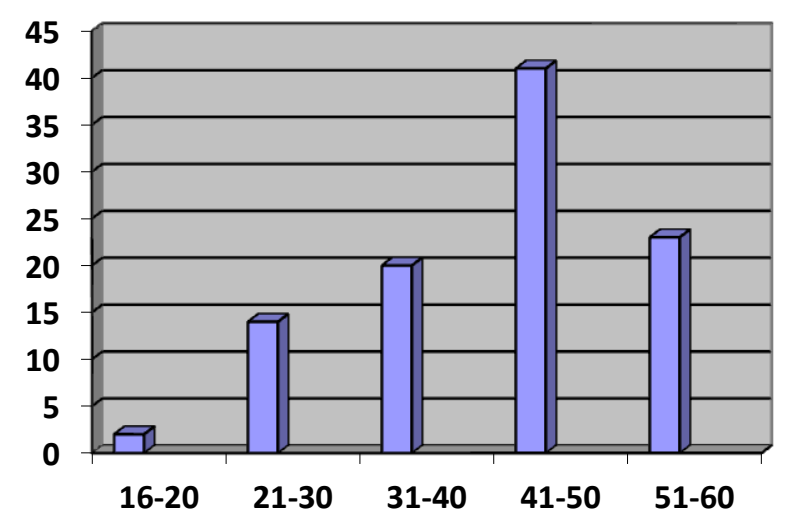

2) Out of 100 patients male patients were 56 and females 44

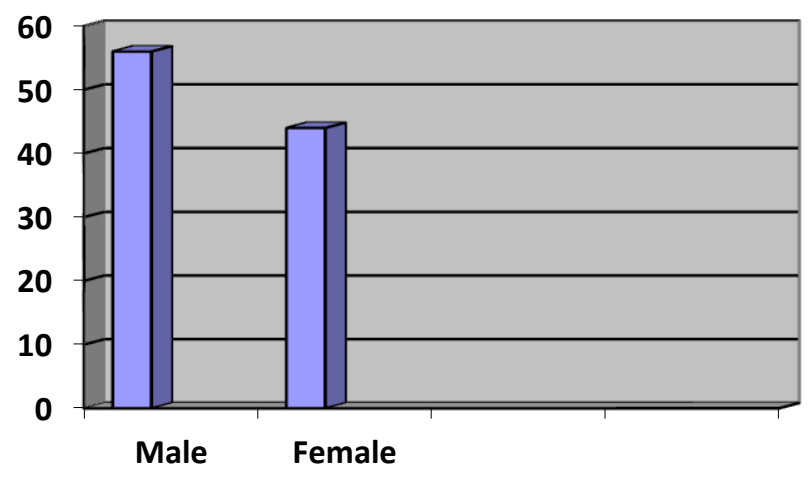

C) Common etiologies are multi organ failure with sepsis $(16 \%)$ followed by snakebite $(12 \%)$, malaria $(10 \%)$, congestive cardiac failure $(9 \%)$, 
urinary tract obstruction (8\%), cholera (6\%), myocardial infarction (6\%), dengue (5\%), Acute exacerbation of COPD and acute gastroenteritis (4\%), while remaining etiologies constituted $20 \%$ of etiological factors.

\begin{tabular}{|lc|}
\hline Etiology & Frequency \\
\hline Multi-organ failure with sepsis & 16 \\
\hline Snake bite & 12 \\
\hline Malaria & 10 \\
\hline CCF & 9 \\
\hline Urinary track obstruction & 8 \\
\hline Myocardial infarction & 6 \\
\hline Cholera & 6 \\
\hline Dengue & 5 \\
\hline Acute gastroenteritis & 4 \\
\hline COPD & 4 \\
\hline Cirrhosis & 3 \\
\hline GI blood loss & 3 \\
\hline Ischemic CVA & 3 \\
\hline Burns & 2 \\
\hline Tuberculosis & 2 \\
\hline Urethral stricture & 3 \\
\hline Glomerulonephritis & 1 \\
\hline Prostatomegaly with UTI & 1 \\
\hline BPH & 1 \\
\hline Viral hepatitis & 1 \\
\hline Total & 100 \\
\hline
\end{tabular}

\section{Discussion}

Acute kidney injury is a common, often under recognized disorder that is increasing in incidence globally, and strongly associated with increase long term morbidity and mortality ${ }^{14}$. The sequelae of AKI are severe and characterized by increased risk of short term and long term mortality, incident chronic kidney disease (CKD) and accelerated progression to end stage renal disease(ESRD) ${ }^{15}$. The in hospital mortality rate is approximately $20 \%$ to $50 \%$ and may exceed $75 \%$ in critically ill patients or patients with sepsis.

In the present study majority of patients $(41 \%)$ were in the age group of 41-50years. The male female ratio is $1.27: 1$. This is similar to the study conducted by Sharma S.K et. $\mathrm{al}^{16}$.

The most common etiological factors of AKI in present study were multi-organ failure with sepsis (16\%), snake bite(12\%), malaria (10\%), CCF $(9 \%)$, urinary tract obstruction $(8 \%)$, cholera $(6 \%)$, myocardial infection $(6 \%)$, dengue $(6 \%)$, acute gastroenteritis (4\%), $\operatorname{COPD}(4 \%)$ while remaining etiologies constituted $20 \%$ of the etiological factors. These findings were almost similar to study of Sharma S.K. et. $\mathrm{al}^{16}$ in which the most common etiological factors were septicemia followed by acute gastroenteritis and congestive cardiac failure. These findings were also almost similar to study by Rebic et. al $^{17}$ in which common etiology factors were acute interstitial nephritis followed by congestive cardiac failure and sepsis.

\section{Conclusion}

Acute kidney injury is an increasingly common and potentially catastrophic complication. Mortality rates remain unacceptably high despite significant advance in health care. The diagnosis of AKI is based on indirect measures of GFR, plasma urea and creatinine concentrations. AKI can be prevented if detected early and appropriate treatment is initiated early in the course of the disease.

\section{References}

1. Brady HR, Brenner BM. Acute renal failure. Harrison's principles of internal medicine. Vol 2. $18^{\text {th }}$ edition. New York: McGraw- Hill: 2005

2. Thadani R, Pascual M, Bonvette VB. Acute renal failure. The New York England journal of Medicine 1996 ; 30 : $1448-1451$

3. Acute renal failure: Definition and Pathogenesis Nissenson AR et.al. Kidney Int Suppl. 1998 May ; 66 : S7-10

4. Chertow GM, Levy EM, Hammermeister $\mathrm{KE}$ et al. Independent association between acute renal failure and mortality following cardiac surgery. Amj Med 104: 343 - 348, 1998

5. Levy EM, Viscoli CM, Horwitz RI. The effect of acute renal failure on mortality : a cohort analysis JAMA 1996; $275: 1489$ 1494

6. Uchino S, Bellomo R, Goldsmith D et. al. An assessment of RIFLE criteria for acute renal failure in hospitalized patients. 
Critical care Med 2006; 34 : 1913 - 1917

7. Mehta RL, Kellum JA, Shah SV, et. al. 2007. Acute Kidney Injury Network (AKIN): report of an initiative to improve outcomes in acute kidney injury. Critical care, 11, R 31

8. Moltoris BA, Levin A, Warnock D, et. al. Improving outcomes of acute kidney injury: report of initiative. /Nat CLIN Pract Nephrol 2007; 3 (8): 439 - 442

9. Kidney Disease : Improving Global Outcomes. Clinical practice guideline on acute kidney injury 2011. www.kidgo.org

10. Mindell JA, Chertow GM. A practical approach to acute renal failure 1997; 81 : $731-747$

11. Hou SH, Broc ME. Hospital acquired renal deficiency. A prospective study. American journal of medicine 1983; $74: 243-24$

12. Hou Sh, Bushinsky DA, Wish JB, Cohen JJ, Harrington JT. Hospital acquired insuffiency : a prospective study. Am J Med 1983; $74: 243-8$

13. Grubb AO. (2000) Cystatin C - properties and use as a diagnostic marker, Adv Clin. Che ; $35 ; 63-99$

14. Lewington AJ, Cerda J,Mehta RL.Raising awareness of acute kidney injury global prespective of a silent killer.Kidney international 2013;84:457-67

15. Harrison's principle of Internal Medicine. $19^{\text {th }}$ edition: $1799-1809$.

16. Sharma S.K.,Prakash Shende, V.B Vikhe, et al. clinical profile of acute kidney in intensive care unit in tertiary care center. Indian Journal of Basic and Applied Medical Reasearch 2014;3(2):511-516

17. Rebic D, Hamzic-Mehmedbasic A, Balavac M, et al. Clinical Analysis of Etiology, Risk Factors and Outcome in patients with Acute Kidney Injury. Material Socio-Medica 2015;27(2);70-74. 